\title{
Hierarchical Space-Time Block Code Recognition Using Correlation Matrices
}

\author{
Vincent Choqueuse, Koffi Yao, Ludovic Collin, and Gilles Burel
}

\begin{abstract}
The blind recognition of communication parameters is a key research issue for commercial and military communication systems. The results of numerous investigations about symbol timing estimation, modulation recognition as well as identification of the number of transmitters have been reported in the literature. But, to our knowledge, none of them have dealt with the recognition of the Space-Time Block Codes (STBC) used in multiple transmitter communications. In order to blindly recognize the STBC of a wireless communication, this paper proposes a method based on the space-time correlations of the received signals. Under perfect timing synchronization and under ideal conditions (full rank channel and a number of receivers greater or equal to the number of transmitters), it shows that the Frobenius norms of these statistics present non-null values whose positions only depend on the STBC at the transmitter side. A classifier for the space-time code recognition of 5 linear STBC (Spatial Multiplexing, Alamouti Coding, and 3 Orthogonal STBC using 3 antennas) is presented. Simulations show that the proposed method performs well even at low signal-to-noise ratios.
\end{abstract}

Index Terms-MIMO, space-time coding, electronic warfare.

\section{INTRODUCTION}

$\mathbf{T}$ HE Blind recognition of communication parameters is an intermediate step between signal detection and signal decoding/demodulation with applications in both commercial and military communication systems. The development of self-configurating receivers lies among civilian applications, whereas military ones include electronic warfare, surveillance and threat analysis. Some algorithms devoted to the blind recognition of symbol timing and frequency offset [1] [2], modulation [3] [4] [5] and propagation channel [6] from the received signals are available in the literature for Single-Input Single-Output (SISO) communications. These data can be used to blindly identify the communication protocol or standard when it is unknown at the receiver side.

Other investigations conducted in parallel have been devoted to the development of new technologies aimed at enhancing the reliability of data transmission in wireless communication systems. Among them, one of the most promising relies on

Manuscript received April 6, 2007; revised August 13, 2007, December 17, 2007, and March 13, 2008; accepted May 8, 2008. The associate editor coordinating the review of this paper and approving it for publication was $\mathrm{K}$. B. Lee. This work is supported by the French "Delegation Generale pour l'Armement" (DGA). The authors would like to thank Ali Mansour, MariePaule Friocourt, and the reviewers for their precious advises.

V.Choqueuse is with the laboratory $E^{3} I^{2}$, EA 3876, ENSIETA, 29806 Brest Cedex 9, France (e-mail: vincent.choqueuse@gmail.com).

K. Yao, L.Collin, and G. Burel are with the laboratory Lab-STICC, FRE CNRS 3167, UBO, CS 93837, 29238 Brest Cedex 3, France. E-mail: KoffiClement (e-mail: \{Yao, Ludovic.Collin, Gilles.Burel\}@univ-brest.fr).

Digital Object Identifier 10.1109/TWC.2008.070364. the use of Multiple-Input Multiple-Output (MIMO) systems in association with Space-Time Codes (STC) [7]. STC is a set of practical signal design techniques aimed at approaching the information theoretic capacity of MIMO channels. The class of linear space-time block codes (STBC) is the major category of space-time codes and can be divided into subclasses like Linear Dispersion Codes [8], Orthogonal STBC [9] [10] and quasi Orthogonal STBC [11]. Currently, some algorithms can blindly recognize the number of transmitters [12] and the modulation [13] of MIMO systems without preliminary knowledge about the propagation channel. Other algorithms are devoted to the blind decoding/demodulation of the received signals [6] [14] [15] [16] [17] [18] [19] and [20] under the assumption that the STBC is known at the receiver side. However, the blind recognition of STBC remains a research issue poorly addressed in the literature.

The method proposed in this paper is aimed at blindly recognizing linear STBC without a priori knowledge about the number of transmitters, the modulation of the transmitted symbols and the propagation channel. The only assumption lies on the perfect estimation of the timing synchronization (one sample per symbol, optimum sampling time) and on the properties of the propagation channel (full rank and with a number of receivers greater or equal to the number of transmitters). Section II of this article presents the signal model of the communication. In section III, a characterization of the STBC based on the Frobenius norms of two spacetime correlations is presented. It is shown that these norms present peaks at certain time lags whose positions only depend on the STBC. Using this characterization, the section IV presents a decision tree classifier for the blind recognition of 5 linear STBC. Finally, the section V summarizes the proposed algorithm and the section VI reports on the performances.

\section{SignAL-MODELS}

Let us denote matrices by boldface capital letters (e.g. M), matrix conjugates, transposes and hermitians by superscripts ${ }^{*},{ }^{T}$ and ${ }^{H}$ respectively (e.g. $\mathbf{M}^{*}, \mathbf{M}^{T}$ and $\mathbf{M}^{H}$ ). Let us also note column matrices by capital letters with a subscript index corresponding to the column number (e.g. $M_{i}$ ) and each matrix element by a capital letter with two subscript indices corresponding respectively to the row and column number (for example $M_{i j}$ ).

\section{A. Signal model of Linear Space-Time block codes}

For any communication using linear STBC, the symbols to be transmitted are sent in block of $n_{e}$ symbols. A block of $n_{e}$ symbols is transmitted to a space-time block encoder 


$$
\begin{aligned}
& \mathbf{C}(S)=\left[C_{0}(S) \cdots C_{l-1}(S)\right]
\end{aligned}
$$

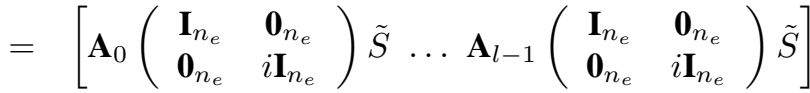

to generate $n_{t}$ parallel signal sequences of length $l$. These sequences are transmitted through $n_{t}$ transmit antennas simultaneously in $l$ time periods. Let us denote by the column vector $S=\left[s_{1}, \cdots, s_{n_{e}}\right]^{T}$, a block of $n_{e}$ complex symbols to be transmitted. Let us also note by $\mathbf{C}(S)$ and $C_{j}(S)(0 \leq j<l)$ the transmission matrix and the $(j+1)^{t h}$ column of this matrix respectively. Under these notations the transmission matrix of a linear space-time block code can be expressed under the general form (1), where $\tilde{S}=\left[\Re e\left(s_{1}\right), \cdots, \Re e\left(s_{n_{e}}\right), \Im m\left(s_{1}\right), \cdots, \Im m\left(s_{n_{e}}\right)\right]^{T}$ denotes the concatenation of the real and imaginary parts of $S$ and where $\mathbf{I}_{n_{e}}$ is the identity matrix of size $n_{e}$. The coding matrices $\mathbf{A}_{j}$ only depend on the STBC. For example, the coding matrices $\mathbf{A}_{0}$ and $\mathbf{A}_{1}$ of an Alamouti code (see appendix C) are defined by:

$$
\begin{aligned}
\mathbf{A}_{0} & =\left(\begin{array}{cccc}
1 & 0 & 1 & 0 \\
0 & 1 & 0 & 1
\end{array}\right) \\
\mathbf{A}_{1} & =\left(\begin{array}{cccc}
0 & -1 & 0 & 1 \\
1 & 0 & -1 & 0
\end{array}\right)
\end{aligned}
$$

In this paper, the symbols $s$ are assumed to belong to the same complex linear modulation $(\geq 4 P S K, \geq 4 Q A M)$ and to be independent and identically distributed (i.i.d). For a complex modulation, the real and imaginary parts of the transmitted symbols are i.i.d so the symbols $s$ respect the following condition:

$$
E\left[\Re e(s)^{2}\right]=E\left[\Im m(s)^{2}\right]=\frac{E\left[|s|^{2}\right]}{2}
$$

\section{B. Signal model of the communication}

Let us consider a receiver composed of $n_{r}$ antennas which is assumed to be perfectly synchronized with the emitter (one sample per symbol, optimum sampling time). In a non cooperative environment, the start and the length of the spacetime block code are both unknown at the receiver side. Let us consider without loss of generality that the first received column, denoted by $Y_{0}$, intercepts the $\left(k_{1}+1\right)^{t h}$ column $\left(0 \leq k_{1}<l\right)$ of the $j^{t h}$ transmitted block, denoted $C_{k_{1}}\left(S_{j}\right)$. Under these assumptions, the $k^{t h}$ intercepted column, $Y_{k}$ $(k \geq 0)$, is described by the following signal model:

$$
\begin{aligned}
Y_{k} & =\mathbf{H} X_{k}+B_{k} \\
\text { with } X_{k} & =C_{u}\left(S_{v}\right)
\end{aligned}
$$

where $u=\left(k+k_{1}\right) \bmod l, v=j+\left(k+k_{1}\right) \operatorname{div} l$, and $x \operatorname{div} l$, $x$ mod $l$ denote respectively the quotient and the remainder of the division $\frac{x}{l}$. $\mathbf{H}$ corresponds to the $n_{r} \times n_{t}$ channel matrix which is composed of i.i.d. complex Gaussian entries (Rayleigh channel) and $B_{k}$ is a vector of size $n_{r}$ representing the additive noise. The additive noise is supposed to be
Gaussian complex circular and temporally uncorrelated.

Our aim being the blind recognition of the space-time block code of a communication from $N$ received samples $Y_{k}(0 \leq$ $k<N)$, an intuitive way to do it is to measure the space and time redundancy of the transmitted symbols with space-time correlations.

\section{Characterization by the Frobenius nORM OF SPACE-TIME CORRELATIONS}

\section{A. Analysis of the space-time correlations}

In order to assess the space-time redundancy of a random complex vector $M_{k}$, we define the two space-time correlations, $\mathbf{R}_{M, T}(\tau)$ and $\mathbf{R}_{M, H}(\tau)$ respectively given by:

$$
\begin{aligned}
\mathbf{R}_{M, T}(\tau) & =\lim _{N \rightarrow \infty} \frac{1}{N} \sum_{k=0}^{N-1} E\left[M_{k} M_{k+\tau}^{T}\right] \\
\mathbf{R}_{M, H}(\tau) & =\lim _{N \rightarrow \infty} \frac{1}{N} \sum_{k=0}^{N-1} E\left[M_{k} M_{k+\tau}^{H}\right]
\end{aligned}
$$

Note that these two correlations only differ by the term $M_{k+\tau}^{T}$ and $M_{k+\tau}^{H}$. Let us consider the space-time correlation of the transmitted samples $X(k)$. In the following, we only develop the theoretical expression of the correlation $\mathbf{R}_{X, T}(\tau)$ as $\mathbf{R}_{X, H}(\tau)$ can be obtained in a similar way. According to the equation (6), the correlation $\mathbf{R}_{X, T}(\tau)$ can be expressed with respect to the structure of the Space-Time Code as (9). Using the signal model of the equation (1), this correlation is equal to:

$$
\begin{aligned}
\mathbf{R}_{X, T}(\tau) & =\lim _{N \rightarrow \infty} \frac{1}{N} \sum_{u=k_{1}}^{N+k_{1}-1}\left[\mathbf{A}_{u \bmod l}\left(\begin{array}{ll}
\mathbf{I}_{n_{e}} & \mathbf{0}_{n_{e}} \\
\mathbf{0}_{n_{e}} & i \mathbf{I}_{n_{e}}
\end{array}\right)\right. \\
& \times E\left[\left(\tilde{S}_{j+u \operatorname{div} l}\right)\left(\tilde{S}_{j+(u+\tau) \operatorname{div} l}^{T}\right)\right] \\
& \left.\times\left(\begin{array}{ll}
\mathbf{I}_{n_{e}} & \mathbf{0}_{n_{e}} \\
\mathbf{0}_{n_{e}} & i \mathbf{I}_{n_{e}}
\end{array}\right) \mathbf{A}_{(u+\tau) \bmod l}^{T}\right]
\end{aligned}
$$

The symbols to be transmitted are independent and identically distributed and respect the equation (4), so the expectation term can be expressed as:

$$
\begin{aligned}
E\left[\left(\tilde{S}_{j+u \operatorname{div} l}\right)\left(\tilde{S}_{j+(u+\tau) \operatorname{div} l}^{T}\right)\right] & =\delta(u \operatorname{div} l-(u+\tau) \operatorname{div} l) \\
& \times \frac{E\left[|s|^{2}\right]}{2} \mathbf{I}_{2 n_{e}}
\end{aligned}
$$

where $\delta(x)$ denotes the Kronecker delta which is equal to 1 for $x=0$ and 0 for $x \neq 0$. The Kronecker delta reflects the fact that the symbols coming from two different blocks are independent. Let us decompose $u$ under the form $u=v l+w$ where $0 \leq w<l$. Using this decomposition and the equation 


$$
\mathbf{R}_{X, T}(\tau)=\lim _{N \rightarrow \infty} \frac{1}{N} \sum_{u=k_{1}}^{N+k_{1}-1} E\left[C_{u \bmod l}\left(S_{j+u \operatorname{div} l}\right) C_{(u+\tau) \bmod l}^{T}\left(S_{j+(u+\tau) \operatorname{div} l}\right)\right]
$$

$$
\begin{aligned}
\mathbf{R}_{X, T}(\tau) & =\lim _{N \rightarrow \infty} \frac{E\left[|s|^{2}\right]}{2 N}\left[\sum_{w=k_{1}}^{l-\tau-1} \mathbf{A}_{w}\left(\begin{array}{cc}
\mathbf{I}_{n_{e}} & \mathbf{0}_{n_{e}} \\
\mathbf{0}_{n_{e}} & -\mathbf{I}_{n_{e}}
\end{array}\right) \mathbf{A}_{w+\tau}^{T}\right. \\
& +n_{b} \sum_{w=0}^{l-\tau-1} \mathbf{A}_{w}\left(\begin{array}{cc}
\mathbf{I}_{n_{e}} & \mathbf{0}_{n_{e}} \\
\mathbf{0}_{n_{e}} & -\mathbf{I}_{n_{e}}
\end{array}\right) \mathbf{A}_{w+\tau}^{T} \\
& \left.+\sum_{w=0}^{N-\left(n_{b}+1\right) l+k_{1}-1} \mathbf{A}_{w}\left(\begin{array}{cc}
\mathbf{I}_{n_{e}} & \mathbf{0}_{n_{e}} \\
\mathbf{0}_{n_{e}} & -\mathbf{I}_{n_{e}}
\end{array}\right) \mathbf{A}_{w+\tau}^{T}\right]
\end{aligned}
$$

(11) into the equation (10) lead to (12), where $n_{b}=(N-$ $\left.\left(l-k_{1}+1\right)\right)$ div $l$ denotes the number of entire received blocks and where the first and the last sums reflect the fact that the first and last received blocks are not entire. For $N \rightarrow \infty$, the influence of the non-whole blocks is not significant over the number of entire received blocks. So, asymptotically $n_{b}$ can be approximated ${ }^{1}$ by $n_{b} \simeq \frac{N}{l}$ and so:

$$
\mathbf{R}_{X, T}(\tau) \simeq \frac{E\left[|s|^{2}\right]}{2 l} \sum_{w=0}^{l-\tau-1} \mathbf{A}_{w}\left(\begin{array}{cc}
\mathbf{I}_{n_{e}} & \mathbf{0}_{n_{e}} \\
\mathbf{0}_{n_{e}} & -\mathbf{I}_{n_{e}}
\end{array}\right) \mathbf{A}_{w+\tau}^{T}
$$

In the same manner, we can demonstrate that the space-time correlation $\mathbf{R}_{Y, H}(\tau)$ is approximately equal to:

$$
\mathbf{R}_{X, H}(\tau) \simeq \frac{E\left[|s|^{2}\right]}{2 l} \sum_{w=0}^{l-\tau-1} \mathbf{A}_{w} \mathbf{A}_{w+\tau}^{H}
$$

At the transmitter side, the equations (13) and (14) show that the space-time correlations only depend on the structure of the code and on the modulation. At the receiver side, the space-time correlation $\mathbf{R}_{Y, T}(\tau)$ and $\mathbf{R}_{Y, H}(\tau)$ are given from equations (7), (5) and (8), (5) respectively:

$$
\begin{aligned}
\mathbf{R}_{Y, T}(\tau) & =\mathbf{H R}_{X, T}(\tau) \mathbf{H}^{T} \\
\mathbf{R}_{Y, H}(\tau) & =\mathbf{H R}_{X, H}(\tau) \mathbf{H}^{H}+\mathbf{R}_{B, H}(0) \delta(\tau) \mathbf{I}_{n_{r}}
\end{aligned}
$$

These correlations depend on the channel matrix $\mathbf{H}$, on the noise correlation $\mathbf{R}_{B, H}(0)$ and on the correlations at the transmitter side. In the next section, we propose a characterization of the space-time coding based on the Frobenius norm of the space-time correlations $\mathbf{R}_{Y, T}(\tau)$ and $\mathbf{R}_{Y, H}(\tau)$.

\section{B. Analysis of the Frobenius norm}

The Frobenius norm of a matrix $\mathbf{M}$ of size $n \times m$, denoted by $\|\mathbf{M}\|_{F}^{2}$, is defined as:

$$
\|\mathbf{M}\|_{F}^{2}=\sum_{i=1}^{n} \sum_{j=1}^{m}\left|M_{i j}\right|^{2}
$$

Let us consider the two Frobenius norms of the space-time correlations $\left\|\mathbf{R}_{Y, T}(\tau)\right\|_{F}^{2}$ and $\left\|\mathbf{R}_{Y, H}(\tau)\right\|_{F}^{2}$. According to the

\footnotetext{
${ }^{1}$ When the receiver intercepts an entire number of blocks, we obtain the equality on equation (13)
}

equations (15) and (16), it can be proved that:

Proposition 1: For a full rank channel matrix $\mathbf{H}$ of size $n_{r} \times n_{t}\left(n_{r} \geq n_{t}\right)$ and for $\tau>0$,

$$
\begin{array}{lll}
\left\|\mathbf{R}_{Y, T}(\tau)\right\|_{F}^{2}=0 & \text { if and only if } & \left\|\mathbf{R}_{X, T}(\tau)\right\|_{F}^{2}=0(18) \\
\left\|\mathbf{R}_{Y, H}(\tau)\right\|_{F}^{2}=0 & \text { if and only if } & \left\|\mathbf{R}_{X, H}(\tau)\right\|_{F}^{2}=0(19)
\end{array}
$$

Proof: See appendices A and B for proof.

As the probability to obtain a rank-deficient Rayleigh channel with i.i.d entries is small, we propose to recognize the STBC from the positions $\tau$ of the non-null values of $\left\|\mathbf{R}_{Y, T}(\tau)\right\|_{F}^{2}$ and $\left\|\mathbf{R}_{Y, T}(\tau)\right\|_{F}^{2}$. In fact under the assumption that $n_{r} \geq n_{t}$, the proposition 1 and the equations (13) and (14) show that the positions of the non-null values provide information on the length $l$ of the STBC since the Frobenius norms are null for $\tau \geq l$. Furthermore, depending on the coding matrices $\mathbf{A}_{j}$, the norms are also null for certain $\tau<l$. In the following, an automatic classifier for the STBC recognition based on the position of the non-null values of $\left\|\mathbf{R}_{Y, T}(\tau)\right\|_{F}^{2}$ and $\left\|\mathbf{R}_{Y, H}(\tau)\right\|_{F}^{2}$ is exposed.

\section{Classification of Space-Time Code By Decision TREE AND PEAK DETECTOR}

In this section, we propose a classifier for the blind recognition of 5 linear STBC using 2 or 3 transmit antennas. The STBC under consideration are:

1) Spatial Multiplexing (SM) using $n_{t}=2$ transmit antennas; one should note that, though Spatial Multiplexing does not transmit space-time redundancy, it can be interpreted as in the case of a linear STBC.

2) Alamouti coding using $n_{t}=2$ transmit antennas. This code is an Orthogonal STBC of rate 1.

3) $1^{\text {st }}$ Orthogonal $S T B C$ with rate $\frac{3}{4}$ using $n_{t}=3$ transmit antennas.

4) $2^{\text {nd }}$ Orthogonal $S T B C$ with rate $\frac{3}{4}$ using $n_{t}=3$ transmit antennas.

5) Orthogonal $S T B C$ with rate $\frac{1}{2}$ using $n_{t}=3$ transmit antennas. 
TABLE I

VALUes of $\left\|\mathbf{R}_{X, T}(\tau)\right\|_{F}^{2}$ AND $\left\|\mathbf{R}_{X, H}(\tau)\right\|_{F}^{2}$ With RESPECT TO THE SPACE-TIME CODING $\left(E\left[|s|^{2}\right]=1\right)$.

\begin{tabular}{|c|r|c|c|}
\hline Code & $\tau$ & $\left\|\mathbf{R}_{X, T}(\tau)\right\|_{F}^{2}$ & $\left\|\mathbf{R}_{X, H}(\tau)\right\|_{F}^{2}$ \\
\hline SM & 0 & 0 & $n_{t}$ \\
& $>0$ & 0 & 0 \\
\hline \multirow{3}{*}{ Alamouti } & 0 & 0 & 2 \\
& 1 & 0.5 & 0 \\
\hline \multirow{3}{*}{$S T B C 3$} & 1 & 0 & 0 \\
\hline \multirow{3}{*}{ Code 1 } & 1 & 0.25 & 1.6875 \\
& 2 & 0.125 & 0.0625 \\
& 3 & 0 & 0.0625 \\
$\frac{3}{4} S T B C 3$ & 0 & 0 & 0.0625 \\
& 1 & 0.125 & 0 \\
Code 2 & 2 & 0.125 & 0.125 \\
& 3 & 0.125 & 0.0625 \\
& $>3$ & 0 & 0 \\
& 0 & 0 & 0 \\
\hline \multirow{5}{*}{$S T B C 3$} & 1 & 0.0313 & 0.625 \\
Code 3 & 2 & 0 & 0 \\
& 3 & 0.1563 & 0 \\
& 4 & 0.75 & 0.125 \\
& 5 & 0.1563 & 0 \\
& 6 & 0 & 0 \\
& 7 & 0.0313 & 0 \\
& 7 & 0 & 0 \\
\hline \multirow{5}{*}{. } & & & \\
& & &
\end{tabular}

These codes are presented in appendix C. The automatic classification of these STBC from the non-null values of $\left\|\mathbf{R}_{Y, T}(\tau)\right\|_{F}^{2}$ and $\left\|\mathbf{R}_{Y, H}(\tau)\right\|_{F}^{2}$ is performed by a decision tree.

\section{A. Classification by Decision Tree}

At the transmitter side, the theoretical norms of $\left\|\mathbf{R}_{X, T}(\tau)\right\|_{F}^{2}$ and $\left\|\mathbf{R}_{X, T}(\tau)\right\|_{F}^{2}$ are obtained thank's to equations (13) and (14). For the 5 STBC under consideration, these norms are reported on table I. According to the proposition 1, we propose to classify the space-time coding with the decision tree of the figure 1. First, the Orthogonal STBC3 with rate $\frac{1}{2}$ is recognized by a non-null value of the Frobenius norm at $\tau=4$. Then, the presence of the $2^{\text {nd }}$ and $1^{\text {st }}$ Orthogonal STBC3 with rate $\frac{3}{2}$ are detected from a non-null value of the Frobenius norm at $\tau=3$ and $\tau=2$ respectively. Finally, the norm at $\tau=1$ is used to discriminate Alamouti Coding and Spatial Multiplexing. At each node of the tree, the nullity of $\left\|\mathbf{R}_{Y, T}(\tau)\right\|_{F}^{2}$ is tested. In practise, $\mathbf{R}_{Y, T}(\tau)$ is estimated and the test of nullity is performed by a hypothesis testing.

\section{B. Detection of null values using hypothesis test}

In practise, the space-time correlation $\mathbf{R}_{Y, T}(\tau)$ is unknown and assessed from the circular convolution $(\tau=$ $\{0, \cdots, N / 2\})$ :

$$
\widehat{\mathbf{R}}_{Y, T}(\tau)=\frac{1}{N} \sum_{i=0}^{N-1} Y_{i} Y_{(i+\tau) \bmod N}^{T}
$$

The detection of the null values of $\left\|\widehat{\mathbf{R}}_{Y, T}(\tau)\right\|_{F}^{2}$ can be formulated as the following hypothesis test.

- Hypothesis $\mathcal{H}_{0}:\left\|\widehat{\mathbf{R}}_{Y, T}(\tau)\right\|_{F}^{2}=0$.

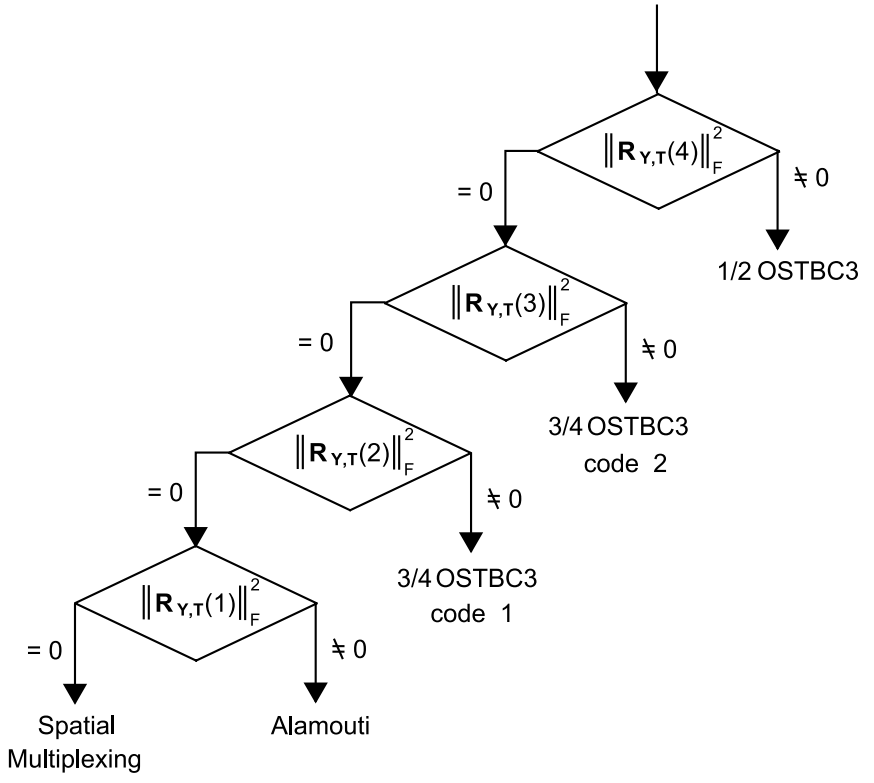

Fig. 1. Decision tree for the recognition of 5 STBC.

- Hypothesis $\mathcal{H}_{1}:\left\|\widehat{\mathbf{R}}_{Y, T}(\tau)\right\|_{F}^{2} \neq 0$.

We propose to apply a preprocessing to adapt, under the hypothesis $\mathcal{H}_{0}$, the statistic $\widehat{\mathbf{R}}_{Y, T}\left(\tau_{0}\right)$ to a chi-square distribution. Let us consider the set of time lag elements, denoted by $\Omega$, for which the theoretical value $\mathbf{R}_{Y, T}\left(\tau_{0}\right)=\mathbf{0}$ when $\tau_{0} \in \Omega$ for any STBC. For the space-time codes of interest, the table I shows that $\Omega=\{8,9,10, \cdots\}$. Let us denote by $\operatorname{vec}\left\{\widehat{\mathbf{R}}_{Y, T}(\tau)\right\}$, the column vector of size $n_{r}^{2}$ which is constructed by concatenating the $n_{r}$ columns of $\widehat{\mathbf{R}}_{Y, T}(\tau)$. According to the central limit theorem, vec $\left\{\widehat{\mathbf{R}}_{Y, T}(\tau)\right\}$ is an asymptotically normal estimator of $\operatorname{vec}\left\{\mathbf{R}_{Y, T}(\tau)\right\}$ and $\operatorname{so} \operatorname{vec}\left\{\widehat{\mathbf{R}}_{Y, T}\left(\tau_{0}\right)\right\} \longrightarrow \mathcal{N}_{c}\left(0_{n_{r}^{2}}, \Psi\right)$ where $0_{n_{r}^{2}}$ is a column vector composed of $n_{r}^{2}$ null elements and where $\Psi=E\left[\operatorname{vec}\left\{\widehat{\mathbf{R}}_{Y, T}\left(\tau_{0}\right)\right\}\left(\operatorname{vec}\left\{\widehat{\mathbf{R}}_{Y, T}\left(\tau_{0}\right)\right\}\right)^{H}\right]$ is a covariance matrix of size $n_{r}^{2} \times n_{r}^{2}$. In practise, this covariance matrix can be estimated by:

$$
\widehat{\Psi}=\frac{1}{|\Omega|} \sum_{\tau_{0} \in \Omega} \operatorname{vec}\left\{\widehat{\mathbf{R}}_{Y, T}\left(\tau_{0}\right)\right\} \operatorname{vec}\left\{\widehat{\mathbf{R}}_{Y, T}\left(\tau_{0}\right)\right\}^{H}
$$

where $|\Omega|$ denotes the number of elements of $\Omega$. Then, a diagonalization of the covariance matrix $\widehat{\Psi}$ is performed to obtain $n_{r}^{2}$ decorrelated gaussian laws. The diagonalization is achieved by applying to $\operatorname{vec}\left\{\widehat{\mathbf{R}}_{Y, T}(\tau)\right\}$ a whitening matrix $\mathbf{W}$ of size $n_{r}^{2} \times n_{r}^{2}$ verifying:

$$
\operatorname{vec}^{d}\left\{\widehat{\mathbf{R}}_{Y, T}(\tau)\right\}=\mathbf{W} \times \operatorname{vec}\left\{\widehat{\mathbf{R}}_{Y, T}(\tau)\right\}
$$

with $E\left[\operatorname{vec}^{d}\left\{\widehat{\mathbf{R}}_{Y, T}\left(\tau_{0}\right)\right\}\left(\operatorname{vec}^{d}\left\{\widehat{\mathbf{R}}_{Y, T}\left(\tau_{0}\right)\right\}\right)^{H}\right]=2 \times \mathbf{I}_{n_{r}^{2}}$

where $\mathbf{I}_{n_{r}^{2}}$ is the identity matrix of size $n_{r}^{2}$. A whitening matrix $\mathbf{W}$ can be extracted from the covariance $\widehat{\Psi}$. Let us consider the eigenvalues decomposition of the covariance matrix defined as $\widehat{\Psi}=\mathbf{U} \Lambda \mathbf{U}^{H}$ where $\Lambda$ and $\mathbf{U}$ correspond respectively to the diagonal matrix which contains the eigenvalues and to the unitary matrix which contains the associated eigenvectors. The whitening matrix is given by:

$$
\mathbf{W}=\sqrt{2} \Lambda^{-1 / 2} \mathbf{U}^{H}
$$




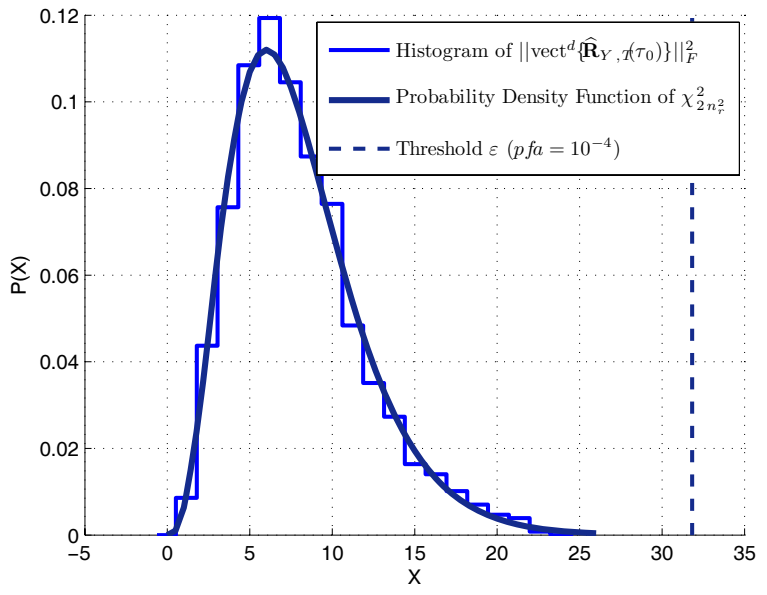

Fig. 2. Distribution of the Frobenius norm of the space time correlation for $\tau \in \Omega$ at SNR=0dB. Communication using Alamouti coding and QPSK modulation. The interceptor is composed of 2 antennas and receives 2048 samples.

After the diagonalization the equation (23) shows that each element of $\operatorname{vec}^{d}\left\{\widehat{\mathbf{R}}_{Y, T}\left(\tau_{0}\right)\right\}$ follows asymptotically a gaussian circular law with zero mean and variance 2 which implies that the square modulus follows a chi-square law with 2 degree of freedom $\chi_{2}^{2}$. As the gaussian elements are also decorrelated, the $n_{r}^{2}$ chi-square laws are independent and so the Frobenius norm of $\operatorname{vec}^{d}\left\{\widehat{\mathbf{R}}_{Y, T}\left(\tau_{0}\right)\right\}$ follows asymptotically a chi-square law with $2 n_{r}^{2}$ degree of freedom, i.e $\left\|\operatorname{vec}^{d}\left\{\widehat{\mathbf{R}}_{Y, T}\left(\tau_{0}\right)\right\}\right\|_{F}^{2} \longrightarrow \chi_{2 n_{r}^{2}}^{2}$.

The knowledge of the distribution of $\left\|\operatorname{vec}^{d}\left\{\widehat{\mathbf{R}}_{Y, T}\left(\tau_{0}\right)\right\}\right\|_{F}^{2}$ under assumption $\mathcal{H}_{0}$ permits one to set the peak detector threshold. The peak detector threshold, $\varepsilon$, is obtained from the probability of false alarm, $p f a$, defined by:

$$
p f a=\int_{\varepsilon}^{\infty} \frac{x^{\frac{(v-2)}{2}}}{2^{v / 2} \Gamma(v / 2)} \mathrm{e}^{-x / 2} d x
$$

where $v$ is the degree of freedom which is equal to $2 n_{r}^{2}$, and $\Gamma()$ is the gamma function such as:

$$
\Gamma(z)=\int_{0}^{\infty} x^{z-1} e^{-x} d x
$$

Taking the inverse function of equation (25) leads to $\varepsilon$. Finally, the hypothesis $\mathcal{H}_{0}$ is chosen if $\left\|\operatorname{vec}^{d}\left\{\widehat{\mathbf{R}}_{Y, T}(\tau)\right\}\right\|_{F}^{2}<\varepsilon$. If the norm is greater than the threshold $\varepsilon$, the hypothesis $\mathcal{H}_{1}$ is chosen.

Figure 2 presents the histogram of $\left\|\operatorname{vec}^{d}\left\{\widehat{\mathbf{R}}_{Y, T}\left(\tau_{0}\right)\right\}\right\|_{F}^{2}$ for a communication using Alamouti coding. The probability density function of the chi-2 law with $2 n_{r}^{2}$ degrees of freedom and the threshold, $\varepsilon$, obtained with a probability of false alarm equal to $10^{-4}$ are plotted on the same figure. It is worth noting that the histogram is well approximated by the chi-2 law. Figure 3 depicts the threshold $\varepsilon$ and the norm $\left\|\operatorname{vec}^{d}\left\{\widehat{\mathbf{R}}_{Y, T}(\tau)\right\}\right\|_{F}^{2}$ as a function of $\tau$ for the same communication. We can remark that the value of the Frobenius norm is greater than $\varepsilon$ only for $\tau=1$. The use of the result of the peak detector together with the decision tree

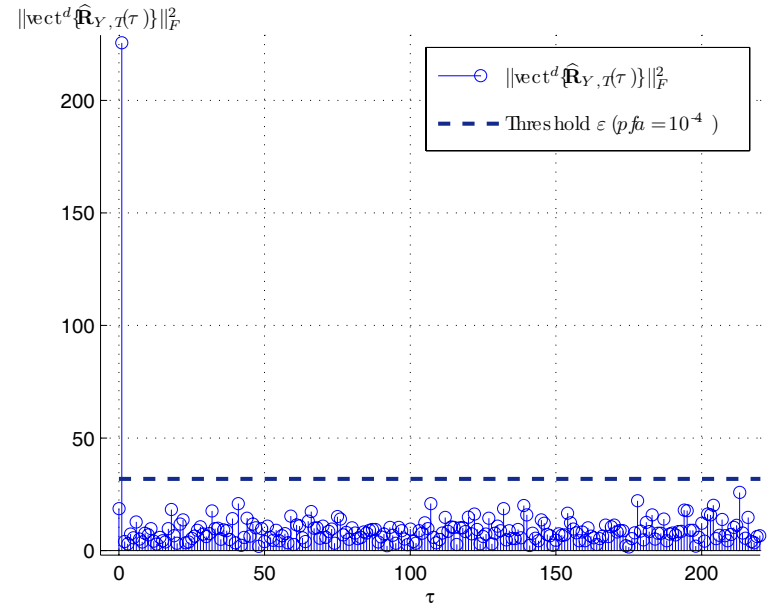

Fig. 3. Threshold of the peak detector with $S N R=0 d B$. Communication using Alamouti coding and QPSK modulation. The interceptor is composed of 2 antennas and receives 2048 samples.

of the figure I allows us to conclude that Alamouti coding is used at the transmitter side.

\section{AlgORITHM SUMMARY}

The proposed STBC recognition algorithm is composed of the following steps:

1) Computation of the time-lag correlation, $\widehat{\mathbf{R}}_{Y, T}(\tau)$ with equation (20) for $\tau=\{0, \cdots, N / 2\}$.

2) Computation of the covariance matrix $\widehat{\Psi}$ of $\operatorname{vec}\left\{\widehat{\mathbf{R}}_{Y, T}\left(\tau_{0}\right)\right\}$ with equation (21) for $\Omega=\{8,9, \cdots, N / 2\}$.

3) Computation of the eigenvalues decomposition of $\widehat{\Psi}$.

4) Computation of the whitened matrix $\mathbf{W}$ from equation (24) and of $\operatorname{vec}^{d}\left\{\widehat{\mathbf{R}}_{Y, T}(\tau)\right\}$ with equation (22).

5) Computation of the Frobenius norm $\left\|\operatorname{vec}^{d}\left\{\widehat{\mathbf{R}}_{Y, T}(\tau)\right\}\right\|_{F}^{2}$.

6) Computation of the peak detector threshold, $\varepsilon$, from the probability of false alarm with equation (25). The degree of freedom of the chi-2 law is equal to $2 n_{r}^{2}$.

7) Classification with the decision tree of the figure 1.

\section{Simulation Results}

Monte Carlo simulations were run to highlight the behavior of the proposed algorithm in different environments. They were aimed at recognizing communications using Spatial Multiplexing, Alamouti coding or Orthogonal STBC3 with rate $\frac{3}{4}\left(1^{\text {st }}\right.$ and $2^{\text {nd }}$ code) and $\frac{1}{2}\left(3^{r d}\right.$ code).

One thousand of Monte Carlo trials was performed for each type of communication. Moreover, the conditions for each Monte Carlo trial were: i) A Rayleigh distributed channel, which means that $H_{u v}$ follows a complex gaussian circular law of unit variance, i.e. $E\left[\left|H_{u v}\right|^{2}\right]=1$, ii) a complex gaussian circular and spatially uncorrelated noise, verifying $\mathbf{R}_{B, H}(0)=$ $\sigma^{2} \mathbf{I}_{n_{r}}$. iii) $S N R=10 \log _{10}\left(\frac{\sigma_{s}^{2}}{\sigma^{2}}\right)$ where $\sigma_{s}^{2}=\operatorname{tr}\left[\mathbf{R}_{X, H}(0)\right]$ and $\operatorname{tr}[$ ] denotes the trace function. 


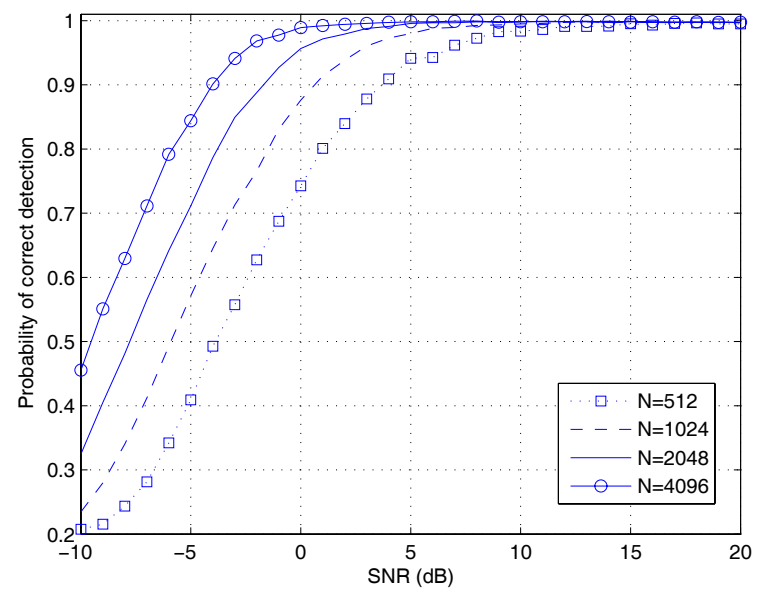

Fig. 4. Effect of the number of received samples $N$ on the probability of correct recognition. Recognition of communications using spatial multiplexing or Alamouti coding and QPSK modulation. The interceptor is composed of 3 antennas. The probability of false alarm is equal to $10^{-4}$.

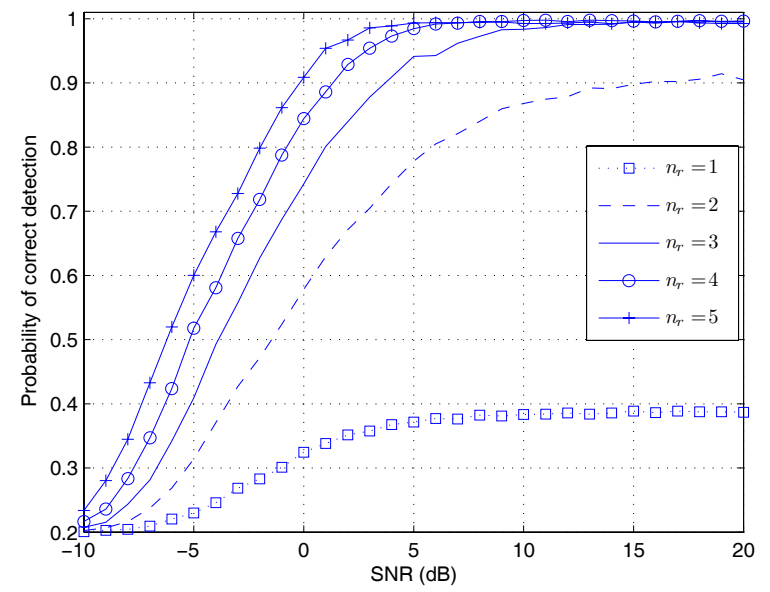

Fig. 5. Effect of the number of receiver antennas $n_{r}$ on the probability of correct recognition. The interceptor receives 512 samples. The probability of false alarm is equal to $10^{-4}$.

\section{A. Influence of the number of received samples $N$}

Figure 4 illustrates the recognition behavior for an interceptor composed of 3 antennas and a number of received samples equals to 512, 1024, 2048 and 4096. In the four cases, the probability of recognition is close to 1 at a SNR equals to $10 \mathrm{~dB}$. The recognition is enhanced by a greater number of received samples. With $N=4096$, the probability of correct detection is close to 1 for a SNR equals to $0 \mathrm{~dB}$. This improvement is due to a better estimate of the correlation matrix $\widehat{\mathbf{R}}_{Y, T}(\tau)$.

\section{$B$. Influence of the number of receiver antennas $n_{r}$}

Figure 5 presents the probability of correct detection with respect to the number of receiver antennas for an interceptor receiving 512 samples and a probability of false alarm equals to $10^{-4}$. It is increased by elevating the number of receiver antennas. For 5 receiver antennas and a SNR close to $5 \mathrm{~dB}$, the probability of correct recognition is about 1 .

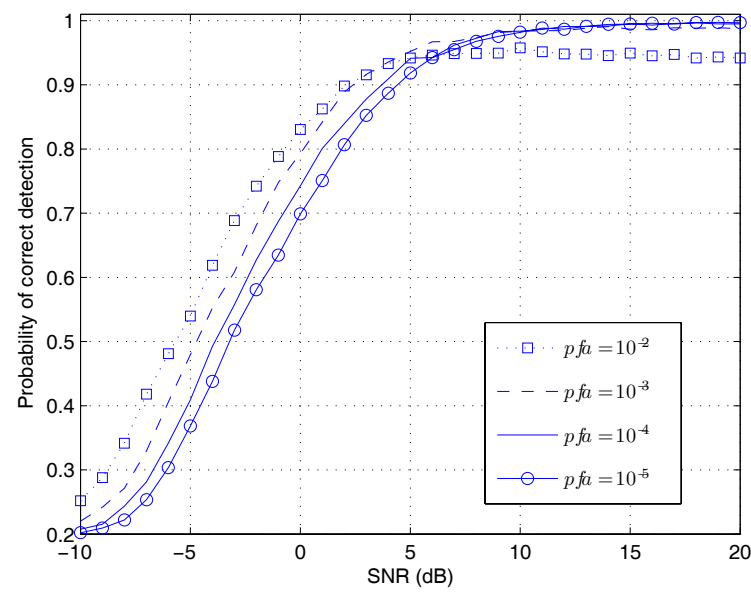

Fig. 6. Effect of the the probability of false alarm $p f a$. Recognition of 5 STBC communications using QPSK modulation. The interceptor is composed of 3 antennas and receives 512 samples.

TABLE II

CONFUSION MATRIX. PROBABILITY OF FALSE ALARM $10^{-2}$. THE INTERCEPTOR IS COMPOSED OF 3 ANTENNAS AND RECEIVES 512 SAMPLES. $S N R=20 d B$.

\begin{tabular}{|c|ccccc|}
\hline In & STC3 $^{(3)}$ & STC3 $^{(2)}$ & STC3 $^{(1)}$ & Alam. & SM \\
\hline STC3 $^{(3)}$ & 1000 & 0 & 0 & 0 & 0 \\
STC3 $^{(2)}$ & 62 & 938 & 0 & 0 & 0 \\
STC3 $^{(1)}$ & 57 & 28 & 915 & 0 & 0 \\
STC2 $_{\text {SM }}^{32}$ & 15 & 15 & 36 & 917 & 0 \\
SM & 16 & 19 & 11 & 939 \\
\hline
\end{tabular}

In fact, a larger number of receiver antennas enhances the amplitude of the Frobenius norm peaks $\left\|\operatorname{vec}^{d}\left\{\widehat{\mathbf{R}}_{Y, T}(\tau)\right\}\right\|_{F}^{2}$ and the discrimination between non-null and null amplitudes is easier. It's interesting to note that in the underdetermined case $\left(n_{r}<n_{t}\right)$, the probability of correct detection is not equal to 1 even for high SNR conditions. For a receiver composed of 1 antenna, this probability is close to 0.4 at $10 \mathrm{~dB}$. In fact in the underdetermined case, the proposition 1 is not valid since $n_{r}<n_{t}$. Increasing the probability of false alarm (pfa) leads to better performances in this case. However with a high false alarm, the risk of detecting a peak where there isn't one increases.

\section{Influence of the probability of false alarm}

Figure 6 depicts the performances of correct recognition versus the probability of false alarm for an interceptor receiving 512 samples and composed of 3 antennas. A higher probability of false alarm gives better results at low SNR since the risk of non detection of a peak is decreased (Type II risk). At a SNR equals to 0 , the probability of detection is close to 0.83 for $p f a=10^{-2}$ and close to 0.7 for $p$ fa $=10^{-5}$. However at high SNR, a lower probability of false alarm gives better results since the risk of detecting a peak when there isn't one is lower (Type I risk). A low pfa leads to perfect detection at a $\mathrm{SNR}=20 \mathrm{~dB}$ whereas $p f a=10^{-2}$ leads to a probability of correct detection close to 0.94 for the same SNR. Table II gives the corresponding confusion matrix. As the confusion matrix is lower triangular, it shows that wrong detections occur 


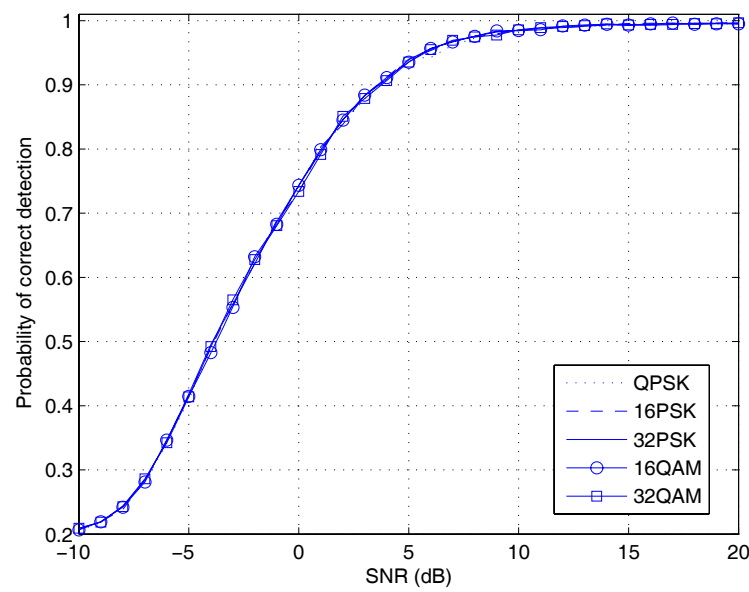

Fig. 7. Effect of the the modulation. Recognition of 5 STBC communications. The interceptor is composed of 3 antennas and receives 512 samples. The probability of false alarm is equal to $10^{-4}$.

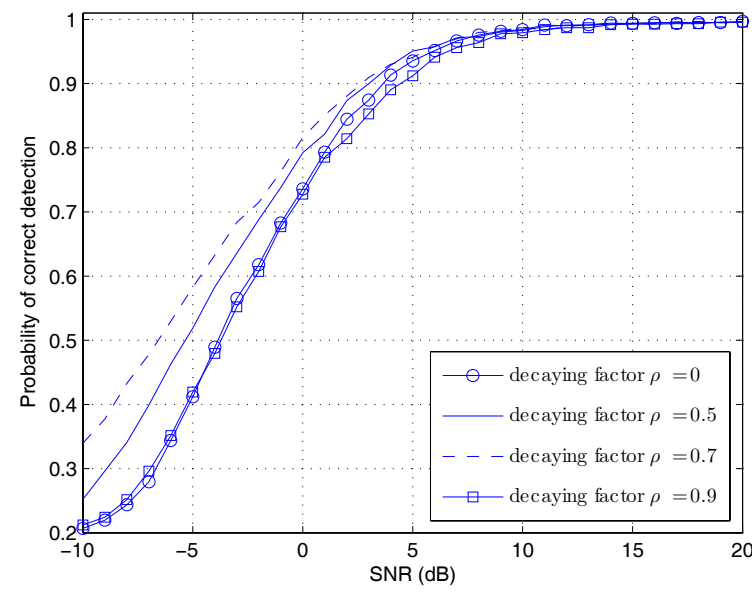

Fig. 8. Effect of spatially correlated noise. Recognition of 5 STBC communications. The interceptor is composed of 3 antennas and receives 512 samples. The probability of false alarm is equal to $10^{-4}$.

when non-null values of the Frobenius norm caused by a bad estimate of $\widehat{\mathbf{R}}_{Y, T}(\tau)$ are detected as peaks (Type I risk).

\section{Influence of the modulation}

We have evaluated the behavior of our algorithm for 4 complex modulations: QPSK, 16PSK, 32PSK, 16QAM and 32QAM. These modulations are mandatory for most of the wireless standards. Figure 7 presents the probability of correct detection with respect to the modulation. The figure shows that the performances of our algorithm do not depend on the complex modulation, the same performances are achieved for the 5 modulations.

\section{E. Influence of spatially correlated noise}

In the previous simulations, the noise is supposed to be spatially and temporally white, i.e the noise covariance matrix is equal to $\mathbf{R}_{B, H}(\tau)=\sigma^{2} \delta(\tau) \mathbf{I}_{n_{r}}$. In the presence of strong spatially colored interference, a more general assumption on

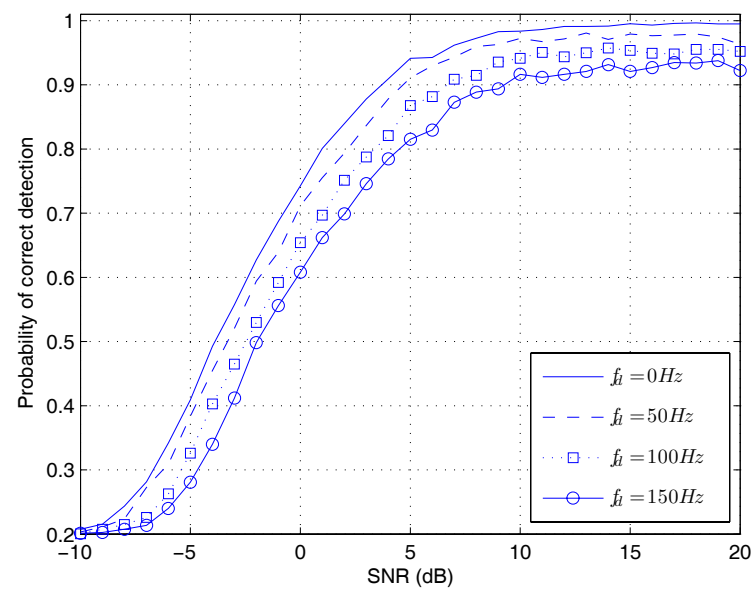

Fig. 9. Effect of the maximum doppler shift of the propagation channel. Recognition of 5 STBC communications using QPSK modulation. The interceptor is composed of 4 antennas and receives 512 samples. The probability of false alarm is equal to $10^{-4}$ and the sample time of the input signal is equal to $10^{-5} \mathrm{~s}$.

the noise term $B(k)$ is that the noise is temporally white but spatially colored [15]. We have evaluated the performances of our algorithm under spatially correlated noise environment. In our simulations, the noise consists of spatially correlated vectors with exponentially decaying correlation among the elements. The element located on the $u^{\text {th }}$ row and the $v^{t h}$ column of the noise covariance matrix $\mathbf{R}_{B, H}(0)$, denoted by $\Xi_{u v}$, is given by:

$$
\Xi_{u v}=\sigma^{2} \rho^{|u-v|} e^{i(u-v) \pi / 2}
$$

where $0 \leq \rho<1$ is a decaying factor. This noise model has been exposed on reference [21]. Theoretically, spatially correlated noise does not influence the performance of our method since we exploit the time lag correlations for $\tau \neq 0$. Figure 8 presents the probability of correct detection versus the factor $\rho$. The figure 8 shows that for a moderate decaying factor $(\rho \leq 0.7)$, the presence of spatially correlated noise improves the probability of correct detection at low SNR. However in a high correlated noise environment $(\rho \geq 0.9)$, the performances decreases.

\section{F. Influence of fast fading channel}

The previous experiments assume that the channel is static, i.e the channel matrix $\mathbf{H}$ does not change over the interception. We have evaluated the performances of our method in the case of a fast fading Rayleigh channel with Jake's doppler spectrum [22]. The figure 9 shows the performances of correct recognition versus the maximum doppler shift, $f_{d}$. In each simulation, the input sample period is fixed to $10^{-5} \mathrm{~s}$. We can remark that the fast fading decreases the probability of correct recognition. For a SNR of $20 \mathrm{~dB}$ and a maximum doppler of $50 \mathrm{~Hz}, 100 \mathrm{~Hz}$ and $150 \mathrm{~Hz}$, the probability of correct detection is respectively equal to $0.97,0.95$ and 0.92 .

\section{G. Influence of synchronization errors}

The application of our STBC blind recognition algorithm requires a perfect estimation of the timing synchronization 


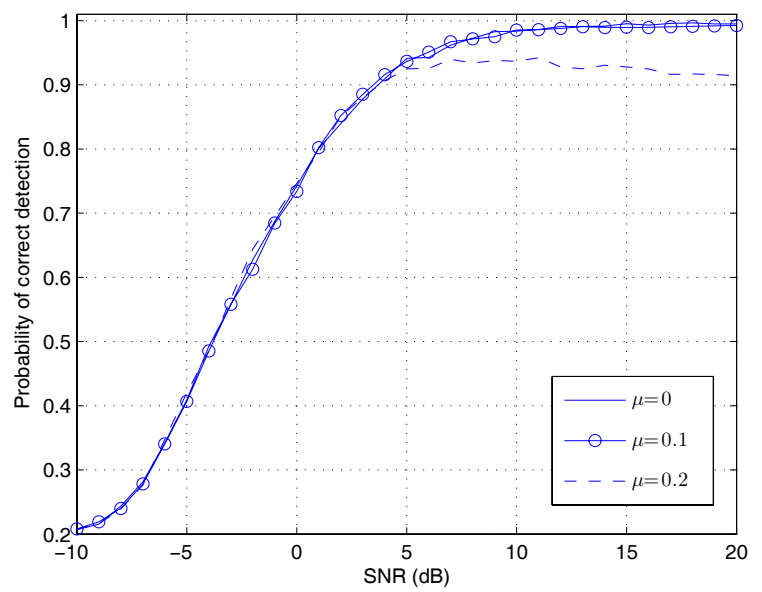

Fig. 10. Effect of the synchronization error $\mu$. Recognition of 5 STBC communications using QPSK modulation. The interceptor is composed of 4 antennas and receives 512 samples. The probability of false alarm is equal to $10^{-4}$.

i.e one sample per symbol and optimum sampling time. We have evaluated the performances of our algorithm with nonoptimum sampling time. In the case of rectangular pulse shapes, sampling time error of $\mu \%$ translates after the matched filtering, to a two-path channel $[1-\mu, \mu]$ [5]. In our simulations, the same error is used for each transmitter-receiver pair. Figure 10 illustrates the behavior of the proposed algorithm for $\mu$ varying from 0 (optimum sampling time) to 0.2 ( $20 \%$ error). For small synchronization error $(\mu=0.1)$, the performances are close to the ones obtained under ideal conditions. However, increasing the synchronization error decreases the probability of correct recognition even for large SNR. For example, we can remark that a synchronization error of $20 \%$ leads to a probability equals to 0.91 at $S N R=20 d B$. In fact, if a peak is present at the time lag $\tau$, sampling time errors introduce new correlation peaks at $\tau-1$ and $\tau+1$. So in the presence of high synchronization errors, the decision tree of figure 1 is not adapted for the recognition of STBC.

\section{CONCLUSION}

This paper described a new method for the blind recognition of STBC under perfect estimation of the timing synchronization. We showed that the Frobenius norm of the space-time correlations exhibits peaks whose positions only depend on the space-time coding. The time lags for which the Frobenius norm is non zero are identified with a peak detector based on hypothesis testing, then the automatic recognition is performed by a decision tree. The performances of our algorithm were evaluated for the recognition of 5 space-time codes using 2 and 3 transmit antennas: Spatial Multiplexing, Alamouti coding and 3 types of Orthogonal STBC3. Experimental results showed a good recognition, even at low SNR and with a limited number of receive antennas. The performances were enhanced by increasing the number of receiver antennas and/or that of received samples. Additional simulations showed that this method still worked with less receivers than transmitters (undertermined case), under spatially correlated noise environments, and with small synchronization errors.

\section{APPENDIX}

\section{A. Proof of the first statement}

Let us demonstrate that:

$$
\left\|\mathbf{R}_{Y, T}(\tau)\right\|_{F}^{2}=0 \quad \text { if and only if }\left\|\mathbf{R}_{X, T}(\tau)\right\|_{F}^{2}=0
$$

under the assumption that the channel matrix $\mathbf{H}$ of size $n_{r} \times n_{t}$ $\left(n_{r} \geq n_{t}\right)$ is full $\operatorname{rank}\left(\operatorname{rank}(\mathbf{H})=\min \left(n_{r}, n_{t}\right)=n_{t}\right)$. The proof requires to demonstrate the two following implications:

$$
\begin{aligned}
\left\|\mathbf{R}_{X, T}(\tau)\right\|_{F}^{2}=0 & \Rightarrow\left\|\mathbf{R}_{Y, T}(\tau)\right\|_{F}^{2}=0 \\
\left\|\mathbf{R}_{Y, T}(\tau)\right\|_{F}^{2}=0 & \Rightarrow\left\|\mathbf{R}_{X, T}(\tau)\right\|_{F}^{2}=0
\end{aligned}
$$

1) Proof of the first implication: Using the equation 15, we obtain the implication:

$$
\begin{aligned}
\left\|\mathbf{R}_{X, T}(\tau)\right\|_{F}^{2}=0 & \Rightarrow \mathbf{R}_{X, T}(\tau)=\mathbf{0}_{n_{t}} \\
& \Rightarrow \mathbf{H R}_{X, T}(\tau) \mathbf{H}^{T}=\mathbf{0}_{n_{r}} \\
& \Rightarrow\left\|\mathbf{H R}_{X, T}(\tau) \mathbf{H}^{T}\right\|_{F}^{2}=0 \\
& \Rightarrow\left\|\mathbf{R}_{Y, T}(\tau)\right\|_{F}^{2}=0
\end{aligned}
$$

2) Proof of the second implication: Using the equation 15, we obtain the implication:

$$
\begin{aligned}
\left\|\mathbf{R}_{Y, T}(\tau)\right\|_{F}^{2}=0 & \Rightarrow \mathbf{R}_{Y, T}(\tau)=\mathbf{0}_{n_{r}} \\
& \Rightarrow \mathbf{H R}_{X, T}(\tau) \mathbf{H}^{T}=\mathbf{0}_{n_{r}} \\
& \Rightarrow \mathbf{H}^{H} \mathbf{H} \mathbf{R}_{X, T}(\tau) \mathbf{H}^{T} \mathbf{H}^{*}=\mathbf{H}^{H} \mathbf{0}_{n_{r}} \mathbf{H}^{*} \\
& \Rightarrow\left(\mathbf{H}^{H} \mathbf{H}\right) \mathbf{R}_{X, T}(\tau)\left(\mathbf{H}^{H} \mathbf{H}\right)^{T}=\mathbf{0}_{n_{t}}(31)
\end{aligned}
$$

For a full rank channel matrix $\mathbf{H}$ of size $n_{r} \times n_{t}\left(n_{r} \geq n_{t}\right)$, the matrix $\mathbf{H}^{H} \mathbf{H}$ is invertible [23]. Multiplying the left and the right of the last equation by $\left(\mathbf{H}^{H} \mathbf{H}\right)^{-1}$ and $\left(\left(\mathbf{H}^{H} \mathbf{H}\right)^{-1}\right)^{T}$ respectively leads to:

$$
\begin{aligned}
\left\|\mathbf{R}_{Y, T}(\tau)\right\|_{F}^{2}=0 & \Rightarrow \mathbf{R}_{X, T}(\tau)=\left(\mathbf{H}^{H} \mathbf{H}\right)^{-1} \mathbf{0}_{n_{t}}\left(\left(\mathbf{H}^{H} \mathbf{H}\right)^{-1}\right)^{T} \\
& \Rightarrow \mathbf{R}_{X, T}(\tau)=\mathbf{0}_{n_{t}} \\
& \Rightarrow\left\|\mathbf{R}_{X, T}(\tau)\right\|_{F}^{2}=0
\end{aligned}
$$

\section{B. Proof of the second statement}

To demonstrate that:

$$
\left\|\mathbf{R}_{Y, H}(\tau)\right\|_{F}^{2}=0 \text { if and only if }\left\|\mathbf{R}_{X, H}(\tau)\right\|_{F}^{2}=0
$$

the proof is quite similar (use equation 16 instead of 15).

\section{STBC examples}

A communication using spatial Multiplexing transmit $n_{t}$ symbols, denoted by the vector $S$, simultaneously via the $n_{t}$ transmit antennas. The transmitted matrix, $\mathbf{C}(S)$, reduces to the following column vector:

$$
(\mathbf{C}(S))^{T}=\left(\begin{array}{lll}
s_{1} & \cdots & s_{n_{t}}
\end{array}\right)
$$

The Alamouti code [9] is an Orthogonal STBC of rate $c=1$ using $n_{t}=2$ transmit antennas. The $v^{t h}$ transmitted matrix, $\mathbf{C}(S)$, is equal to:

$$
(\mathbf{C}(S))^{T}=\left(\begin{array}{cc}
s_{1} & s_{2} \\
-s_{2}^{*} & s_{1}^{*}
\end{array}\right)
$$


An Orthogonal STBC3 of rate $\frac{3}{4}$ using $n_{t}=3$ transmit antennas [7] is defined by the following transmitted matrix $\mathbf{C}(S)$ :

$$
(\mathbf{C}(S))^{T}=\left(\begin{array}{ccc}
s_{1} & 0 & -s_{2}^{*} \\
0 & s_{1} & -s_{3} \\
s_{2} & s_{3}^{*} & s_{1}^{*} \\
-s_{3} & s_{2}^{*} & 0
\end{array}\right)
$$

Another Orthogonal STBC3 of rate $\frac{3}{4}$ using $n_{t}=3$ transmit antennas [7] is defined by the following transmitted matrix $\mathbf{C}(S)$ :

$$
(\mathbf{C}(S))^{T}=\left(\begin{array}{ccc}
s_{1} & s_{2} & s_{3} \\
-s_{2}^{*} & s_{1}^{*} & 0 \\
s_{3}^{*} & 0 & -s_{1}^{*} \\
0 & -s_{3}^{*} & s_{2}^{*}
\end{array}\right)
$$

An Orthogonal STBC3 of rate $\frac{1}{2}$ using $n_{t}=3$ transmit antennas was proposed by Tarokh [10]. The transmitted block, $\mathbf{C}(S)$, is defined by the following matrix:

$$
(\mathbf{C}(S))^{T}=\left(\begin{array}{ccc}
s_{1} & s_{2} & s_{3} \\
-s_{2} & s_{1} & -s_{4} \\
-s_{3} & s_{4} & s_{1} \\
-s_{4} & -s_{3} & s_{2} \\
s_{1}^{*} & s_{2}^{*} & s_{3}^{*} \\
-s_{2}^{*} & s_{1}^{*} & -s_{4}^{*} \\
-s_{3}^{*} & s_{4}^{*} & s_{1}^{*} \\
-s_{4}^{*} & -s_{3}^{*} & s_{2}^{*}
\end{array}\right)
$$

\section{REFERENCES}

[1] F. Gini and G. Giannakis, "Frequency offset and symbol timing recovery in flat fading channels: a cyclostationary approach," IEEE Trans. Commun., vol. 46, no. 3, pp. 400-411, 1998.

[2] S. Lee, "A new non data aided feedforward symbol timing estimator using two samples per symbol," IEEE Commun. Lett., vol. 6, no. 5, pp. 205-207, 1993.

[3] E. Azzouz and A. Nandi, Automatic Modulation Recognition of Communication Signal. Kluwer Academic Publisher, 1996.

[4] O. Dobre, Y. Bar-Ness, and W. Su, "Blind modulation classification: a concept whose time has come," in Proc. IEEE Sarnoff Symposium on Advances in Wired and Wireless Communication, Princeton, NJ, 2005, pp. $223-228$

[5] A. Swami and B. Sadler, "Hierarchical digital modulation classification using cumulants," IEEE Trans. Commun., vol. 48, no. 3, pp. 416-429, 2000 .
[6] C. Chi, C. Chen, C. Feng, and C. Chen, Blind Equalization and System Identification. Springer, 2006.

[7] E. Larsson and P. Stoica, Space-Time Block Coding for Wireless Communication. Cambridge Press, 2003.

[8] B. Hassibi and B. Hochwald, "High-rate codes that are linear in space and time," IEEE Trans. Inform. Theory, vol. 48, no. 7, pp. 1804-1824, 2002.

[9] S. Alamouti, "A simple transmit diversity technique for wireless communication,” IEEE J. Select. Areas Commun., vol. 16, no. 8, pp. 1451-1458, 1998.

[10] V. Tarokh, H. Jafarkhani, and A. Calderbank, "Space time block codes from orthogonal designs," IEEE Trans. Inform. Theory, vol. 45, no. 5, pp. 744-765, 1999.

[11] H. Jafarkhani, "A quasi-orthogonal spacetime block code," IEEE Trans. Commun., vol. 49, no. 1, pp. 1-4, 2001

[12] S. Aouada, A. Zoubir, and S. Cee, "A comparative study on source number detection," in Proc. IEEE ISSPA, vol. 1, Paris, France, 2003, pp. $173-176$

[13] A. Swami, S. Barbarossa, and B. Sadler, "Blind source separation and signal classification," in Proc. Asilomar Conference on Signals, Systems and Computers, vol. 2, Pacific Grove, CA, 2000, pp. 1181-1191.

[14] A. Swindlehurst and G. Leus, "Blind and semi-blind equalization for generalized space-time block codes," IEEE Trans. Signal Processing, vol. 50, no. 10, pp. 2489-2498, 2002.

[15] E. Larsson, P. Stoica, and J. Li, "Orthogonal space-time block codes: maximum likelihood detection for unknown channels and unstructured intereferences," IEEE Trans. Signal Processing, vol. 51, no. 2, pp. 362 $372,2003$.

[16] J. Liu, A. Iserte, and M. Lagunas, "Blind separation of OSTBC signals using ICA neural networks," in Proc. IEEE ISSPIT, Darmstadt, Germany, 2003, pp. 502-505.

[17] S. Shahbazpanahi, A. Gershman, and J. Manton, "Closed form blind MIMO channel estimation for othogonal space-time codes," IEEE Trans. Signal Processing, vol. 53, no. 12, pp. 4506-4517, 2005

[18] W. Ma, B. Vo, T. Davidson, and P. Ching, "Blind ML detection of orthogonal space-time block codes: efficient high-performance implementations," IEEE Trans. Signal Processing, vol. 54, no. 2, pp. 738-751, 2006.

[19] T. Cui and C. Tellambura, "Efficient blind receiver design for orthogonal space-time block codes," IEEE Trans. Wireless Communication, vol. 6, no. 5, pp. 1890-1899, 2007.

[20] L. Zhou, J. Zhang, and K. Wong, "A novel signaling scheme for blind unique identification of alamouti space-time block coded channel," IEEE Trans. Signal Processing, vol. 55, no. 6, pp. 2570-2582, 2007.

[21] P. Stoica and M. Cedervall, "Detection tests for array processing in unknown correlated noise fields," IEEE Trans. Signal Processing, vol. 45, no. 9, pp. 2351-2362, 1997.

[22] M. Ganz, "A power-spectral theory of propagation in the mobile-radio environment," IEEE Trans. Veh. Technol., vol. 21, no. 1, p. 27-38, 1972.

[23] G. Golub and C. V. Loan, Matrix Computations. The John Hopkins University Press, 1996. 